\title{
Classification of Brain MRI Images using Computational Intelligent Techniques
}

\author{
Saurabh Shah \\ Department of Computer Science and Engineering, \\ Babaria Institute of Technology, Vadodara and R.K. \\ University, Rajkot, Gujarat, India
}

\author{
N.C. Chauhan \\ Department of Information Technology, \\ A D Patel Institute of Technology, \\ Anand, Gujarat, India
}

\begin{abstract}
MRI of brain can reveal important abnormalities and brain diseases such as brain tumours if these MRI images can be processed properly by intelligent algorithms. As the MRI images have low contrast and contain noise; it is difficult to precisely separate the region of interest between tumour and normal brain tissues.

In this paper, computationally intelligent techniques have been presented to classify brain MRI images into normal and abnormal (having tumour) ones.

The first method uses Gabor filters to extract the texture features from magnetic resonance brain images and then performs classification between normal and abnormal images using Support Vector Machine (SVM). A second method is also presented which uses novel histogram comparison method of left and right halves of brain based on Bhattacharya coefficient and finds bounding box as region of interest (ROI). Texture features are extracted using Gabor filters from this ROI. Finally the classification of images was performed using Artificial Neural Networks. A comparison of both the proposed methods is given at end.
\end{abstract}

\section{Keywords}

MRI, brain tumour, support vector machine, artificial neural network, Gabor filter, classification, feature extraction

\section{INTRODUCTION}

Some abnormalities in the human brain like haemorrhage and brain tumour require immediate attention and must be diagnosed at the earliest. As per the research of National Brain Tumour Foundation (NBTF) in United States, around 13 thousand people die out of 29 thousand people in the U.S. who are diagnosed with primary brain tumours each year. Also as per the estimation of World Health Organization (WHO), brain tumour is considered to be one of the most common brain diseases, so its diagnosis at early stage and timely treatment can help more than 4 lac affected persons per year in the world to increase chances of survival [1]. To detect presence of tumour, to study its clinical properties and to delineate tumour from healthy tissues, multiple MRI sequences are required which include T1-weighted(T1), T1weighted with contrast enhancement (T1c), T2-weighted(T2) and Fluid Attenuated Inversion Recovery (FLAIR) because one MRI sequence is not sufficient to identify/segment tumour completely and different sequences reveal different information. Moreover, the complexity of detection and identification yet increases since brain tumours reveal different appearance among different patients [2].

T1-weighting is generally used for the structural analysis of brain. It also allows to separate out healthy tissues. In T1weighted contrast-enhanced images (gadolinium-DTPA), the tumour borders exhibit sharp edges and higher intensities which appear brighter in the tumour region. So, the active tumour and necrotic regions can be distinguished easily. In T2-weighted MRI, the edema region appears bright and it is surrounded by the tumour. FLAIR sequence specifically helps to separate out the edema region from the CerebroSpinal Fluid (CSF)[2, 5]. In our experiment 60 MRI images with T1, T1-weighted contrast-enhanced, T2 and FLAIR sequences have been used, where 30 images are of normal brains and another 30 images are of brains that contain tumour.

Texture analysis has been effectively used in many applications in image processing which extracts and quantifies features based on local patterns in images. Literature has shown applications of texture analysis of brain MRI images for the detection of tumours based on their texture properties [3]. It has given promising results in segmenting brain tumours from MRI which has potential to outperform analysis done by experts in terms of diagnostic accuracy [4].

Mainly for medical image processing applications, , segmentation of images based on textural feature methods give more reliable and desired results compared to segmentation based on gray level. Therefore, texture-based analysis is widely used in analysis of medical images [6].

Instead of only measuring mean values in the Region of Interest (ROI), researchers have shown that conventional MRI has higher potential when complicated features are extracted, such as rotation invariant texture features based on Gabor filtering from the central and marginal tumoural, edematous and necrotic region [4].

Micro-patterns of textures like edges, lines, spots and flat areas in an image present different kinds of useful discriminative information [7]. In our experiment, Gabor filters have been applied to extract rotation-invariant texture features from MRI images. Tumours contain texture micropatterns at different scales and orientations. These micropatterns help to discriminate between tumour and normal tissues.

Gabor filters have been applied to compute moments (mean, standard deviation, skewness) based features from the magnitude of Gabor filter responses which helped to extract important features of tumour. Extracted features after applying various Gabor filters are stored in feature vectors.

In our work, classification of MR images into normal and abnormal (having tumour) have been performed using two classifiers - Support Vector Machine, and Artificial Neural Network. Support vector machine (SVM) has proved its applicability and efficiency across wide range of applications 
with promising classification accuracy. It has also been used for detecting and classifying tumours in MR brain images [4, $5,6]$ and for segmentation of brain tumour, edema and necrosis in [5]. The organization of the paper is as follows. Section 2, elaborates the related work that have been reported in the literature. The proposed method with details of feature extraction methods and classifiers have been presented in section 3. Section 4 shows the results of the experiments and their comparison. Finally, section 5 concludes the paper.

\section{RELATED WORK}

Comprehensive review of development in techniques for brain tumour analysis, registration, feature extraction and segmentation methods are presented by Stefan Bauer et. al. They have reviewed state of the art methods for brain tumour analysis with performance measures. Out of major feature extraction techniques, most common features used for the brain tumour segmentation is the image intensities based on different graylevels and local image textures as different tumour areas have different textural patterns. Features based on alignment make use of spatial prior knowledge, obtained either from registration of a standard atlas into the patient image or based on symmetries between left and right brain hemispheres. Intensity gradients or edge-based features could be used for evolving a contour toward the tumour border [2]. To extract the texture features of magnetic resonance (MR) tumour images, Gabor wavelet analysis was carried out to differentiate primary central nervous system lymphoma (PCNSL) and glioblastoma multiforme (GBM) in [6]. Gabor wavelet transform with eight orientations and various frequencies was performed on contrast-enhanced T1-weighted MR images to extract the discriminant features, including tumour shape information. SVM was used for segmentation and a classification model was built based on the extracted features using LDA. Experiments showed that the proposed hybrid method, which used Gabor wavelet analysis, a support vector machine and linear discriminant analysis, could distinguish different diagnosis categories of tumour images [6].

Segmentation is the process of dividing an image into homogeneous regions with similar characteristics such as gray level, colour, texture, brightness, and contrast [19]. Fast Bounding Box (FBB) algorithm proposed by Baidya Nath Saha et. al. was a novel fast segmentation technique which used brain symmetry to find out anomaly like tumours enclosed in a bounding box within an axial brain MR image using Bhattacharya coefficient to compute local histogram. Experimental results of FBB where compared with Intensity based bounding box algorithm (IBB) and Entropy based bounding box algorithm (EBB) and found more promising and accurate. The approach avoided the challenge of dealing with the variation of intensities among different MR image slices. Moreover, FBB did not need image registration. The method was completely unsupervised. It was also very efficient and could be implemented in real time [8]. Detailed review on image segmentation presented by Sharma et. al. has ranked algorithms on the basis of its suitability, applicability, performance and computational cost. As per review, segmentation techniques based on gray level techniques such as region based techniques and thresholding were the simplest techniques and had limited applications. However, by combining them with artificial intelligence techniques, their performance could be improved. Techniques based on textural features which make use of atlas or look-up-table have shown excellent results in medical image segmentation. However, for building the atlas they needed expert's knowledge. Problem with atlas based technique was difficultly to correctly select and label data in some circumstances and also in segmenting complex structure with variable size, shape and properties. Some unsupervised methods such as fuzzy-c-means could be used in these kinds of cases [9]. Neural network-based algorithms could be used for texture-based segmentation and classification but they need heavy supervision and training and their performance depends upon the training method and data used in training [9]. An efficient detection of brain tumour based on wavelet transform, mathematical morphology K-means technique has been applied by Ahmed KHARRAT et. al. [10]. Contrast enhancement in tumour image by processing the mathematical morphology reduced the extraction steps. Wavelet transforms was applied for segmentation and finding the localisation of suspicious regions. Finally, K-means algorithm was used to extract the tumour. Three enhancement algorithms were applied - the well known Beghdadi one, Contrast Limited Adaptive Histogram Equalization algorithm (CLAHE) and Mathematic Morphology. Comparative study of the different algorithms showed that the Mathematic Morphology was better in terms of image enhancement. Results of K- means to extract tumour indicated significant outcome in comparison with expert result [10]. The detection of tumour based on locating the area that breaks the left-right symmetry of the brain was presented in [11]. Block wise asymmetry analysis between left and right region of brain was computed based on Bhattacharya coefficient calculated from normalized histograms with the same range from both parts. The algorithm was tested on 73 images containing tumour, tumour with edema or only edema. The algorithm could detect tumour of varying size correctly in $98.63 \%$ of cases, while it could correctly extract area of tumour in around $71 \%$ of cases. Algorithms didn't aim for precise segmentation of the brain tumour, but only detection of approximate location of the tumour. This location could be then used for more precise tumour extraction, and could make this task easier [11]. Algorithm for automatic edema segmentation in FLAIR images was presented in [12]. This type of contrast FLAIR images was selected because of the visibility and manifestation of edema in this image type. Since in healthy brain, the approximate left-right symmetry generally exists, it was used as the prior knowledge in this paper for searching the approximate edema location. For the detection, asymmetry analysis using Bhattacharya coefficient was used. Since the edemas manifest as a hyperintense area in FLAIR images, it was extracted using thresholding. For the automatic determination of the threshold, the Otsu's algorithm was used. Every image contained a tumour with an edema or only an edema. Various shapes, locations, and sizes of these pathological areas and various image resolutions were tested. Even relatively small edemas were segmented correctly [12].

\section{PROPOSED WORK}

\subsection{Pre-processing}

Pre-processing mainly includes brain extraction, skull removal from MR images and image enhancement. The extraction of brain from skull is applied based on the technique of well-known Chan-Vese algorithm of active contour segmentation method [12].

The Chan-Vese algorithm uses variational calculus methods to evolve the level set function. It performs a mapping that takes a level set function $\varphi$ as input, and returns a real number as shown in Eq. (1). The problem is then to search a function that is a critical point either minimum or maximum. The 
objective is to find out mapping whose critical points are level sets that give useful segmentations for a given problem [12]

For example, suppose we have a bi-level image $I$ with domain that takes on only the values -1 and 1 . Define a functional $F$ such that, for any function $\varphi: \Omega \rightarrow R$

$$
F(\varphi)=\int_{\Omega} H(\varphi) I,
$$

where $H$ is the Heaviside function defined as,

$$
H(x)=\left\{\begin{array}{l}
1, x \geq 0 \\
0, x=0
\end{array}\right.
$$

In the practical, discrete-image case the integral is interpreted as a sum over all the image pixels. The value of this functional is simply the integral of $I$ over all the pixels where $\varphi$ is positive.

Since the level of noise is much lower than the signal of tissues in brain MRI, the computation is not complicated. At first, the points of background are filtered out. The threshold is computed as an average value from the region multiplied by two, where no tissue is present. Since the tissues cannot be present in top corners of the image, the threshold is computed from these parts which is nearly 0 and appears as almost black. The image is then thresholded with this threshold value and the Chan-Vese segmentation algorithm is applied where the boundary surrounding the brain is then extracted.

Image enhancement in medical imaging is performed by applying algorithms so as to make image more presentable for humans or computers and more enhanced in quality which helps to improve segmentation and classification process. It mainly consists of enhancing contrast, removing noise, improving edges and modifying shapes as specified in [10]. For contrast enhancement of brain MRI, histogram normalization has been applied as shown in Eq. (3) where values are normalized in the range of 0 to 255 in our work as it is low contrast image.

$$
I_{N}=(I-\operatorname{Min}) \frac{\text { newMax }- \text { newMin }}{\text { Max }- \text { Min }}+\text { newMin }
$$

\subsection{Extracting Region Of Interest (ROI) using Bhattacharya Coefficient}

Image of detected brain after skull removal is divided into two parts of the same size based on symmetry axis which is parallel to vertical axis. A square block is generated which has side length calculated as one quarter of the longer side of the input image. In this case, given block size can detect both small and large sized tumours. Now, the algorithm is executed in both halves symmetrically by this block. To ensure the overlapping of particular area, the step size is set smaller than the block size. Comparison is done between these areas and their opposite symmetric part. The step size has been set as one sixteenth of the block size.

Comparison is performed by computing Bhattacharya Coefficient (BC) $[24,25]$. From both the symmetric parts, normalized histograms and the Bhattacharya coefficient are computed as follows:

$$
B C=\sum_{i=1}^{N} \sqrt{l(i) \cdot r(i)}
$$

where, $l$ and $r$ represent histograms of blocks in left and right half, respectively and $N$ denotes the total number of bins in the histogram. Bhattacharya coefficient takes values between 0 and 1 , where higher the value, smaller the difference between histograms. For the next computation, the asymmetry is computed as:

$$
A=1-B C
$$

This asymmetry is computed for all blocks. The global maximum of asymmetry from all blocks is detected as described in $[24,25]$. This is the most asymmetric block and most likely to contain the tumour.

\subsection{Feature extraction using Gabor Wavelets}

Because Gabor wavelets capture the local structure corresponding to spatial frequency (scales), spatial localization, and orientation selectivity, they are widely applied in many research areas, such as texture analysis and image segmentation $[3,19,21]$.

A 2D Gabor filter is a product of an elliptical Gaussian in any rotation and a complex exponential representing a sinusoidal plane wave [3, 19]. It is defined as [19]:

$$
\begin{aligned}
& \varphi(x, y, f, \theta)=\frac{f^{2}}{\pi \gamma \eta} e^{-\left(\frac{f^{2}}{\gamma^{2}} x^{\prime 2}+\frac{f^{2}}{\gamma^{2}} y^{\prime 2}\right)} e^{j 2 \pi f x^{\prime 2}} \\
& x^{\prime}=x \cos \theta+y \sin \theta \\
& y^{\prime}=-x \sin \theta+y \cos \theta
\end{aligned}
$$

where $f$ is the central frequency of the sinusoidal plane wave, $\theta$ is the rotation angle of both the Gaussian major axis and the plane wave, $\gamma$ is the sharpness along the major axis, and $\eta$ is the sharpness along the minor axis. The sharpness values along the major axis $\gamma$ and along the minor axis $\eta$ are set to 1 .

Image texture features can be extracted by convolving the image $M(x, y)$ with Gabor filters:

$$
g(x, y ; f, \theta)=M * \varphi(x, y ; f, \theta)
$$

Gabor filters with different frequencies $f_{i}$ and orientations $\theta_{j}$ are selected to obtain the texture features.

frequency, $f_{i}=\frac{\mathrm{f}_{\max }}{(\sqrt{2})^{\mathrm{i}-1}}, i=1,2, \ldots, u$

orientation, $\theta_{j}=\frac{(\mathrm{j}-1) \pi}{\mathrm{v}}, j=1,2, \ldots, v$

After the image is convolved with Gabor wavelets with different frequencies and orientations, the extracted texture features, $\mathrm{G}$, are obtained as [12]:

$$
G=\left\{g_{i j}\left(x, y, f_{i}, \theta_{j}\right) \mid i=1, \ldots, N ; j=0, \ldots, 7\right\}
$$

where ,

$$
g_{i j}\left(x, y ; f_{i}, \theta_{j}\right)=\left\|M * \varphi\left(x, y ; f_{i}, \theta_{j}\right)\right\|
$$

is the magnitude of the Gabor filter response. By averaging the magnitude values of the pixels of the image over all directions, the different frequency features $v_{j}$, which represent 
the texture features of the image, are obtained as $V=\left(v_{0}, v_{l}\right.$ $\left., \ldots, v_{i}, \ldots, v_{N}\right), i=1, \ldots, N$, where $V$ is the texture feature vector of the image.

\subsection{Classification Methods}

\subsubsection{Classification with Support Vector Machine}

Classification in this work is performed using a well-known SVM classifier [15, 18, 30]. SVM is discriminative classifier, originating from machine learning theory. During a training step, it finds a separating hyperplane for the data in the feature space [20]. SVM solves the the following optimization problem:

$$
\min _{w, b, \xi} \frac{1}{2} w^{T} w+C \sum_{i=1}^{l} \xi_{i}
$$

subject to

$$
y_{i}\left(w^{T} \phi\left(x_{i}\right)+b\right) \geq 1-\xi_{i}, \quad \text { with } \xi_{i} \geq 0
$$

where $\left(x_{i} ; y_{i}\right)$ are the instance-label pairs of the dataset and $w$ represents the normal vector of the separating hyperplane. $C$ denotes a penalty parameter for the error and $b$ denotes the offset of the hyperplane. The important property of SVM is that it offers the the use of a kernel function

$$
K\left(x_{i}, x_{j}\right)=\phi\left(x_{i}\right)^{T} \phi\left(x_{j}\right)
$$

for transforming the data into a higher-dimensional feature space, where the data can be linearly separated efficiently with a maximum margin. Slack variables $\xi_{\mathrm{i}}$ are used for softmargin classification [20].

\subsubsection{Classification with Artificial Neural Network}

Neural network applications in computer-aided diagnosis represent one of the main streams of computational intelligence in medical imaging. Because of the adaptive learning of neural networks from input information using a suitable learning algorithm which can also improve themselves based on the varieties of input contents, its applications are seen in many medical imaging problems. It also gives reliable solutions based on given specifications as it has capability of optimising the relationship between the inputs and outputs by learning, and processing [22, 23].

There are several different neural network architectures and learning algorithms available for medical imaging applications, among which one of the most popular is the feed-forward network with backpropagation algorithm [17, $21,22,27,30]$.

In a feed-forward network, the neurons in each layer are only connected with the neurons in the next layer. These connections are unidirectional, which means signals or information being processed can only pass through the network in a single direction, from the input layer, through the hidden layer(s) to the output layer. Feed-forward networks commonly use the back propagation supervised learning algorithm to dynamically alter the weights and bias values for each neuron in the network. The algorithm works by iteratively altering the connection weight values for neurons based on the error in the network's actual output value when compared to the target output value. The actual modification of weights is carried out using a gradient descent algorithm, where the weights are modified after each training example is present to the network [29]. Feed-forward neural network architecture used for our experiment implemented in Matlab environment is shown in Fig. 1.

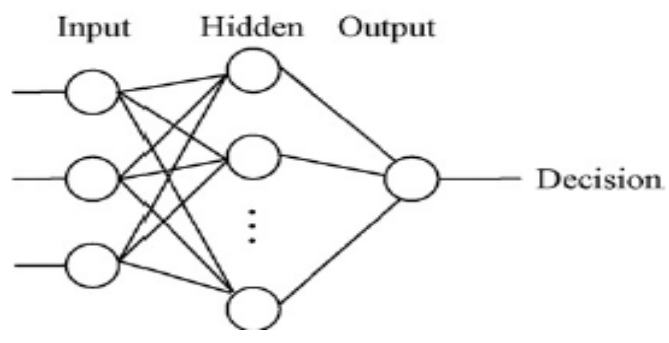

Figure 1: Sample structure of Feed-forward Neural Network with backpropagation used for classification of MRI images

Neural network requires heavy computation when processing high dimensional data. So, to reduce size of an input image and number of dimensions, asymmetry analysis presented in $[24,25]$ is performed based on Bhattacharya coefficient and block containing tumour is identified. Only block expected to contain tumour is given to Neural Network as input image instead of whole brain MRI image.

\subsection{Proposed Methods}

The steps of the two proposed methods used to classify MR brain images into normal and abnormal categories are depicted in Fig.2 and Fig. 3. The detailed description of steps is described in above subsections.

Algorithm (Method 1): Classification of MR brain images using SVM with texture analysis using Gabor Filter

Input: Brain MRI images of different modalities

(T1, T1c, T2, T2FLAIR)

Output: Images labelled with Normal or Abnormal (i.e. with tumour) category

Steps:

1. The brain from MR image is extracted using active contour and thresholding.

2. Contrast Enhancement is performed by Histogram normalization.

3. Texture Feature Extraction is performed by applying Gabor Filters following the steps defined in section 3.3

4. Train SVM classification model using the features extracted in step 3.

5. Extract the features for test images following step 3.

6. Perform the classification of feature vectors extracted from test images using SVM model generated in step 4.

Figure 2: Important steps of the proposed method 1

\section{EXPERIMENTAL RESULTS}

\section{Image database used}

A total of 60 samples of different subjects (persons) and different sequences of MRI including T1, T1c, T2 and FLAIR are used which include normal images and abnormal images. MRI brain images are collected from Brainix - Brain Tumour image collection from Pixmeo [13] (Medical Imaging Software Company), Swiss and National Institutes of Health, USA [14].

All algorithms based on both proposed methodology like Brain extraction using Thresholding, symmetry analysis using Bhattacharya coefficient, feature extraction using Gabor 
Filters and classification using ANN or SVM are implemented in Matlab environment and results are shown below. The results of both of the computational techniques used to classify normal and abnormal images are shown in Table 3.

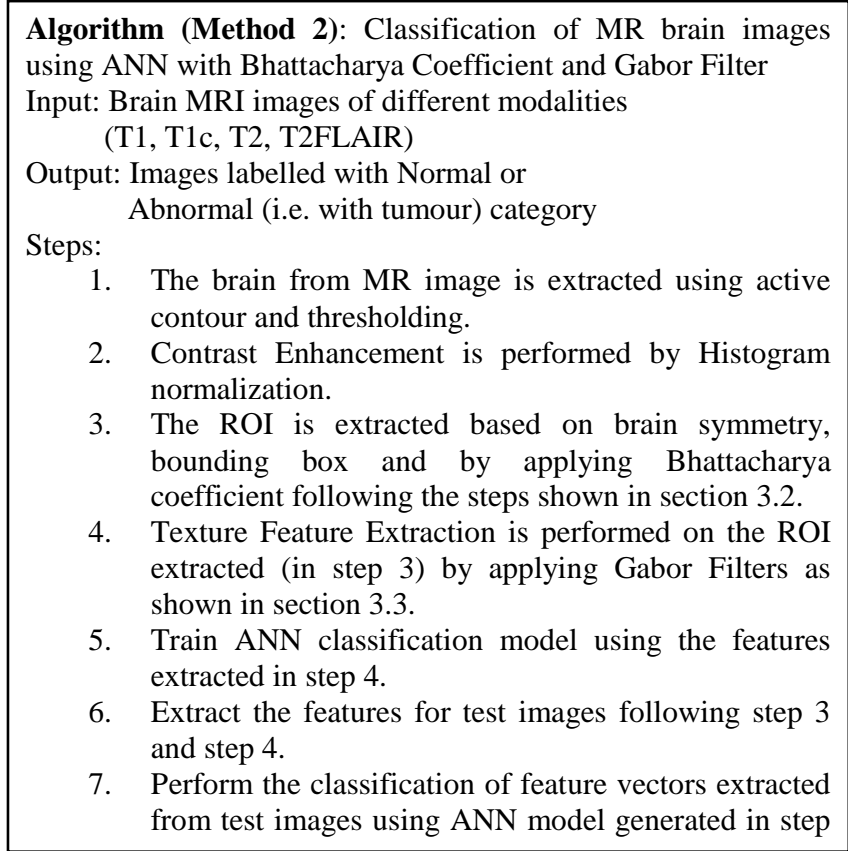

Figure 3: Important steps of the proposed method 2

\section{Brain extraction from skull:}
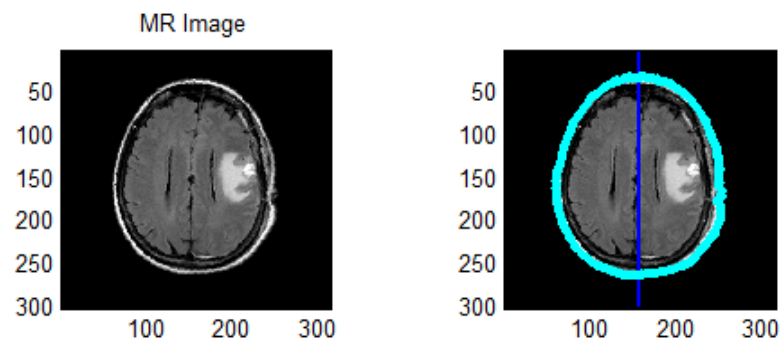

Figure 4: T1C axial brain MRI slice with brain extracted from skull

The Chan-Vese segmentation algorithm is applied where the boundary surrounding the brain is then extracted as shown in Fig. 4 represented by cyan colour. Assuming that the brain is approximately symmetric, the symmetry axis is set to be parallel to the vertical axis and to divide the detected rectangle into two parts of the same size. Blue line indicates symmetry axis.

\section{Extraction of ROI using Bhattacharya Coefficient:}

A sample block with red colour containing possible tumour extracted from the abnormal image based on brain symmetry and as explained in section 3.2 is shown in Fig. 5. Extracted block is resized to $20 * 20$ image size.

\section{Feature extraction using Gabor Wavelets:}

In our experiment, Gabor filters are applied with 8 orientations $(\mathrm{v}=8)$ and 5 scales $(\mathrm{u}=5)$ to extract texture features of MRI images which are stored in feature vectors. So, a total of 40 Gabor filters are applied. Gabor filter window size is set to $4 * 4$ and $\mathrm{f}_{\max }$ is considered as 0.25 in eq. 8.

Fig. 6 shows the Gabor wavelets with five frequencies and eight directions applied on brain MRI in our experiment to extract texture features. The figures show the finer texture features which are detected using higher-frequency Gabor filters.

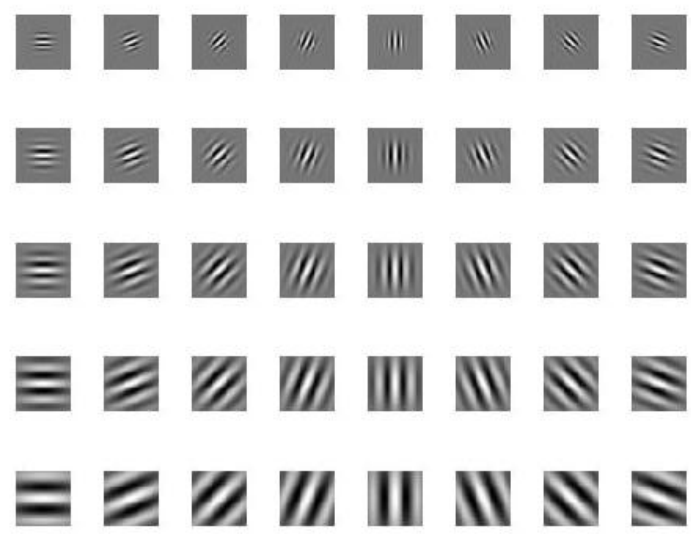

Figure 6: Gabor wavelets in five scales and eight orientations applied on brain MRI

The size of feature vector depends on number of scales and orientations selected as well as on size of image which is calculated as follows.

Size of feature vector $=S^{*} U^{*} V$,

where $U$ is number of scales, $V$ is number of orientations and $\mathrm{S}$ is image size/window size.

So, for our experiment, considering $U=5$ (Scales), $V=8$ (Orientations), image size $=64 * 64$ and window size $=4 * 4$, $S=(64 * 64) /(4 * 4)$

Size of Feature Vector $=S * U * V=[(64 * 64) /(4 * 4)] * 5 * 8=$ 10240 in case of SVM.

Comparison of classification results for both the proposed methods:

Extracted texture features from MRI brain images after applying Gabor filters in proposed method 1 are stored into feature vector which is given to SVM classifier for training and then to classify into normal or abnormal image. Feature vector size is 10240 for input image size of $64 * 64$ Parameter selection for the SVM classifier with different kernel functions is shown in Table 1 and results are obtained using 10 -fold cross-validation.

Extracted texture features from the ROI, after applying Gabor filters in proposed method 2 are stored into feature vector which is given to Feed-forward Neural Network for training and then to classify into normal or abnormal image. All the blocks are resized to $20 * 20$ sizes. Feature vector size is 1000 for input image size of $20 * 20$ as per calculation shown earlier. Parameter selection for ANN is shown in Table 2. 


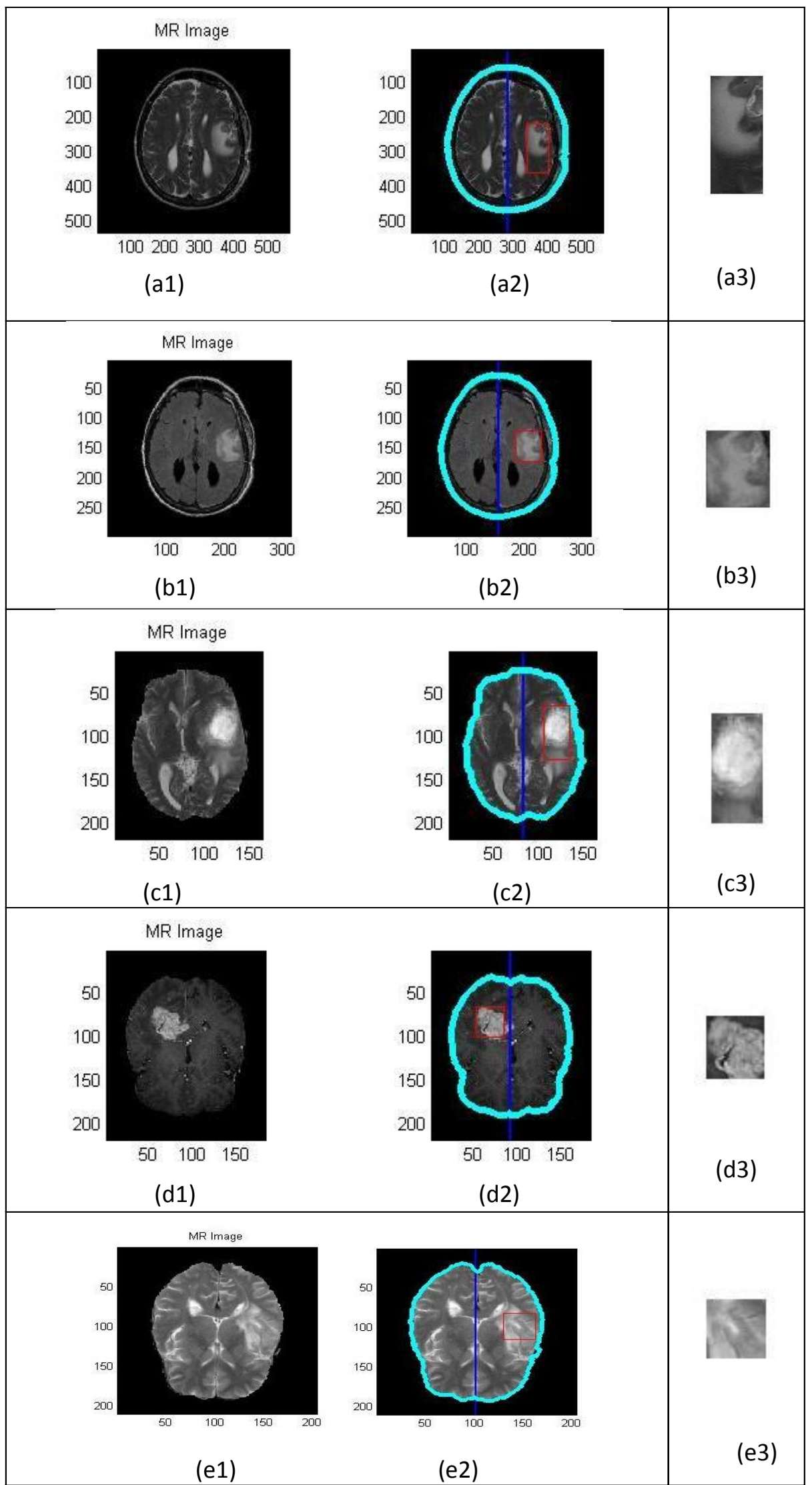

Figure 5: Original Images :( a1),(b1),(c1),(d1),(e1) Extracted Brains: (a2),(b2),(c2),(d2),(e2) ROI block with possible Tumour: $(\mathrm{a3}),(\mathrm{b3}),(\mathrm{c3}),(\mathrm{d} 3),(\mathrm{e} 3)$ 
Classification accuracy, sensitivity and specificity measures are used to evaluate the performance of the proposed methods. Let $T P, T N, F P$, and $F N$ be the numbers of true positive (Normal), true negative (Abnormal), false positive, and false negative samples, respectively.

$$
\begin{gathered}
\text { Accuracy }=\frac{(T P+T N)}{(T P+T N+F P+F N)} \\
\text { Specificity }=\frac{T N}{(T N+F P)} \\
\text { Sensitivity }=\frac{T P}{(T P+F N)}
\end{gathered}
$$

Comparison of two different methods applied on brain MRI images for classification with best results obtained in each method based on parameter selection are summarized in Table 3. In method1, SVM with different kernel functions and different image sizes are experimented while in method 2, Feed-forward neural network with backpropagation algorithm and novel approach of ROI extraction using Bhattacharya Coefficient are applied. Different number of neurons are also experimented at hidden layer to optimize neural network. Results show that ANN with 10 neurons at hidden layer performs better than 5 neurons which is shown in Table 2. In both the methods texture features are extracted using 40 Gabor filters with 5 scales and 8 orientations. Based on comparison of methods, SVM with linear kernel function applied on image size of $64 * 64$ gives highest accuracy $(93.3 \%)$, specificity $(93.3 \%)$ and sensitivity $(93.3 \%)$ compared to other methods as shown in Table 3.

Table 1: Results of Classification of MRI images using Method-1 (SVM with texture analysis using Gabor Filter)

\begin{tabular}{|c|c|c|c|c|c|c|}
\hline $\begin{array}{c}\text { Image } \\
\text { Size }\end{array}$ & $\begin{array}{c}\text { Gabor Filter } \\
\text { parameters }\end{array}$ & $\begin{array}{c}\text { Feature Vector } \\
\text { Size }\end{array}$ & $\begin{array}{c}\text { Parameter } \\
\text { Selection }\end{array}$ & Accuracy & Specificity & Sensitivity \\
\hline $256 * 256$ & $\begin{array}{c}\text { Scale=5, } \\
\text { Orientation=8 }\end{array}$ & 163840 & Linear Kernel & 83.33 & 83.33 & 83.33 \\
\hline $128 * 128$ & $\begin{array}{c}\text { Scale=5, } \\
\text { Orientation=8 }\end{array}$ & 40960 & Linear Kernel & 90 & 90 & 90 \\
\hline $\mathbf{6 4} * \mathbf{6 4}$ & $\begin{array}{c}\text { Scale=5, } \\
\text { Orientation=8 }\end{array}$ & $\mathbf{1 0 2 4 0}$ & Linear Kernel & $\mathbf{9 3 . 3 3}$ & $\mathbf{9 3 . 3 3}$ & $\mathbf{9 3 . 3 3}$ \\
\hline $64 * 64$ & $\begin{array}{c}\text { Scale=5, } \\
\text { Orientation=8 }\end{array}$ & 10240 & $\begin{array}{c}\text { RBF Kernel } \\
\text { Sigma=1) }\end{array}$ & 50 & 46.67 & 53.33 \\
\hline $64 * 64$ & $\begin{array}{c}\text { Scale=5, } \\
\text { Orientation=8 } \\
\text { Scale=5, } \\
\text { Orientation=8 }\end{array}$ & 10240 & $\begin{array}{c}\text { Polynomial } \\
\text { Kernel }\end{array}$ & 50 & 0 & 100 \\
\hline $64 * 64$ & 10240 & MLP Kernel & 63.33 & 60 & 66.67 \\
\hline
\end{tabular}

Table 2: Results of Classification of MRI images using Method-2 (ANN with Bhattacharya Coefficient and Gabor Filter)

\begin{tabular}{|c|c|c|c|c|c|c|c|}
\hline $\begin{array}{c}\text { Image } \\
\text { Size }\end{array}$ & $\begin{array}{c}\text { ROI Size after } \\
\text { applying BC } \\
\text { based } \\
\text { approach }\end{array}$ & $\begin{array}{c}\text { Gabor Filter } \\
\text { parameters }\end{array}$ & $\begin{array}{c}\text { Feature } \\
\text { Vector Size }\end{array}$ & $\begin{array}{c}\text { Number } \\
\text { of } \\
\text { neurons } \\
\text { at hidden } \\
\text { layer }\end{array}$ & Accuracy & Specificity & Sensitivity \\
\hline $\begin{array}{c}256 * \\
256\end{array}$ & $20 * 20$ & $\begin{array}{c}\text { Scale=5, } \\
\text { Orientation=8 }\end{array}$ & 1000 & 5 & $73.3 \%$ & $83.3 \%$ & $63.3 \%$ \\
\hline $\mathbf{2 5 6 *}$ & $\mathbf{2 0 * 2 0}$ & $\begin{array}{c}\text { Scale=3, } \\
\text { Orientation= } \\
\mathbf{2 5 6}\end{array}$ & $\mathbf{5 7 5}$ & $\mathbf{1 0}$ & $\mathbf{8 0} \%$ & $\mathbf{7 0} \%$ & $\mathbf{9 0} \%$ \\
\hline
\end{tabular}

Table 3: Comparison of classification results of both the proposed methods (Method-1 and Method-2) with their optimized parameters

\begin{tabular}{|c|c|c|c|c|c|c|c|c|}
\hline Experiment & $\begin{array}{c}\text { Image } \\
\text { Size }\end{array}$ & $\begin{array}{c}\text { ROI Size } \\
\text { after } \\
\text { applying BC } \\
\text { based } \\
\text { approach }\end{array}$ & $\begin{array}{c}\text { Gabor Filter } \\
\text { parameters }\end{array}$ & $\begin{array}{c}\text { Feature } \\
\text { Vector } \\
\text { Size }\end{array}$ & $\begin{array}{c}\text { Parameter } \\
\text { selection }\end{array}$ & Accuracy & Specificity & Sensitivity \\
\hline Method -1 & $64 * 64$ & - & $\begin{array}{c}\text { Scale=5, } \\
\text { Orientation=8 }\end{array}$ & 10240 & $\begin{array}{c}\text { Linear } \\
\text { Kernel }\end{array}$ & 93.33 & 93.33 & 93.33 \\
\hline Method -2 & $\begin{array}{c}256 * \\
256\end{array}$ & $20 * 20$ & $\begin{array}{c}\text { Scale=3, } \\
\text { Orientation=5 }\end{array}$ & 375 & $\begin{array}{c}\text { Number of } \\
\text { neurons }=10\end{array}$ & $80 \%$ & $70 \%$ & $90 \%$ \\
\hline
\end{tabular}




\section{DISCUSSION AND CONCLUSION}

In this paper, two different computational intelligent methods were presented for automatic classification of MR images into normal and abnormal (with tumour). A set of Gabor filters with five frequencies and eight directions were applied to different modalities of MR images to extract tumour texture features. From results simulation study (Table-2), both methods were found to be comparable, however Method-1 provided quite better accuracy compared to Method-2. It was observed during simulation that Method-1 handled more features extracted from whole image directly while method-2 worked on features of segmented region only. Method-2 was found to be more computationally intensive for high dimensional data generated by extracted features using Gabor filter. Therefore, in method-2 a different approach of histogram comparison using Bhattacharya Coefficient was used for reduction of feature dimensions. However, performance of ANN classifier was dependent on identification of correct block containing tumour. In some cases, block could not identify a tumour correctly and degraded performance of ANN classifier was noticed. Further, more neurons in hidden layer of ANN increased problem of computational complexity. This problem could be solved using efficient feature reduction techniques in future work to reduce dimensions further before giving them as input to ANN. Classification results were acceptable but could be improved further in future work by yet optimizing parameters of both classifiers.

\section{REFERENCES}

[1] N.Rajalakshmi, V.Lakshmi Prabha "Computer-Aided Diagnosis Systems for Brain Pathology Identification Techniques in Magnetic Resonance Images - A Survey", International Journal of Emerging Trends in Engineering and Development, Issue 3, Vol.2, May 2013

[2] Stefan Bauer, Roland Wiest, Lutz-P Nolte and Mauricio Reyes , "A survey of MRI-based medical image analysis for brain tumour studies", Journal of PHYSICS IN MEDICINE AND BIOLOGY, June 2013

[3] Mihran Tuceryan, Anil K. Jain, "Texture Analysis", The Handbook of Pattern Recognition and Computer Vision (2nd Edition), by C. H. Chen, L. F. Pau, P. S. P. Wang (eds.), pp. 207-248, World Scientific Publishing Co., 1998

[4] Evangelia I. Zacharaki, Sumei Wang, Sanjeev Chawla, Dong Soo Yoo, Ronald Wolf, Elias R. Melhem and Christos Davatzikos, "Classification of brain tumour type and grade using MRI texture and shape in a machine learning scheme" Magn Reson Med. December 2009

[5] J. Mikulka and E. Gescheidtov, "An Improved Segmentation of Brain Tumour, Edema and Necrosis", Progress In Electromagnetics Research Symposium Proceedings, Taipei, March, 2013

[6] Yi-hui Liu, Manita Muftah, Tilak Das, Li Bai, Keith Robson, Dorothee Auer, "Classification of MR tumour images based on Gabor wavelet Analysis", Journal of Medical and Biological Engineering , 32(1): 22-28, 2011

[7] Hussain, Muhammad, et al. "Effective extraction of Gabor features for false positive reduction and mass classification in mammography." Appl. Math 8.1L (2014): 397-412.
[8] Baidya Nath Saha, Nilanjan Ray, Russell Greiner, Albert Murtha and Hong Zhang, "Quick detection of brain tumours and edemas: A bounding box method using symmetry", Elsevier ,Computerized Medical Imaging and Graphics 36.2 : 95-107,2012

[9] Sharma, Neeraj, and Lalit M. Aggarwal. "Automated medical image segmentation techniques." Journal of medical physics/Association of Medical Physicists of India 35.1: 3, 2010

[10] Ahmed KHARRAT, Mohamed Ben MESSAOUD, Nacéra BENAMRANE, Mohamed ABID,"Detection of brain tumour in medical images." Signals, Circuits and Systems (SCS), 2009 3rd International Conference on. IEEE, 2009.

[11] Kropatsch, Walter G., Fuensanta Torres, and Geetha Ramachandran. "Detection of Brain Tumours Based on Automatic Symmetry Analysis." 18th Computer Vision Winter Workshop, 2013

[12] Dvorak P., K. Bartusek, and W. G. Kropatsch. "Automated Segmentation of Brain Tumour Edema in FLAIR MRI Using Symmetry and Thresholding.", PIERS Proceedings, Stockholm, Sweden, Aug. 12-15, 2013

[13] MRI Brain Datasets available on Osirix http://www.osirix-viewer.com/datasets/

[14] MRI Brain Datasets available at National Cancer Institute - Research Group under National Institutes of Health (NIH), USAhttps://public.cancerimagingarchive.net/ncia/home.jsf

[15] Zhiqiang Lao, Dinggang Shen, Dengfeng Liu, Abbas F. Jawad, Elias R. Melhem, Lenore J. Launer, R. Nick Bryan, Christos Davatzikos, "Computer-Assisted Segmentation of White Matter Lesions in 3D MR Images Using Support Vector Machine”, Academic Radiology, Vol 15, No 3, March 2008

[16] Ghulam Gilanie , Muhammad Attique , Hafeez-Ullah , Shahid Naweed, Ejaz Ahmed, Masroor Ikram, "Object extraction from T2 weighted brain MR image using histogram based gradient calculation, Pattern Recognition Letters 34,2013

[17] S. Rajasekaran, and G.A.V. Pai, "Neural network, Fuzzy logic, and genetic algorithms - Synthesis and Applications", Prentice Hall India, 2006

[18] N. Cristianini, and J. S.-Taylor, "An introduction to support vector machines and other kernel based learning methods", Cambridge University Press, 2000.

[19] V. Kyrki, J. K. Kamarainen and H. Kalviainen, "Simple Gabor feature space for invariant object recognition," Pattern Recognition Letter, Elsevier, 311-318, 2004.

[20] Stefan Bauer, Lutz-P. Nolte, and Mauricio Reyes, "Fully Automatic Segmentation of Brain Tumour Images Using Support Vector Machine Classification in Combination with Hierarchical Conditional Random Field Regularization", Medical Image Computing and Computer-Assisted Intervention - MICCAI 2011, Springer Berlin Heidelberg, 2011

[21] Neeraj Sharma, Amit K. Ray, Shiru Sharma, K. K. Shukla, Satyajit Pradhan, Lalit M. Aggarwal, "Segmentation and classification of medical images 
using texture-primitive features: Application of BAMtype artificial neural network", Journal of Medical Physics, Jul-Sep; 33(3): 119-126, 2008.

[22] J. Jiang, P. Trundle, J. Ren, "Medical image analysis with artificial neural networks, Elsevier, Medical Imaging and Graphics 34, 617-631,2010

[23] CB, Chan HP, Lin JS, Li H, Freedman MT, Mun SK. Artificial convolution neural network for medical image pattern recognition. Neural Networks 1995, 8(7/8):12011214,1995

[24] Pavel Dvorak, Walter Kropatsch, "Detection of Brain Tumours Based on Automatic Symmetry Analysis", 18th Computer Vision Winter Workshop, Austria, February 46, 2013

[25] Ray, N. and Saha, B.N. and Graham Brown, M.R., "Locating Brain Tumours from MR Imagery Using Symmetry," Signals, Systems and Computers, 2007. ACSSC 2007. Conference Record of the Forty-First Asilomar Conference on, pp. 224-228, November 2007
[26] M. Haghighat, S. Zonouz, M. Abdel-Mottaleb, "Identification Using Encrypted Biometrics," Computer Analysis of Images and Patterns, Springer Berlin Heidelberg, pp. 440-448, 2013.

[27] S. Haykin, Neural network: A comprehensive foundation, (2nd Edition), Prentice Hall India, 2004.

[28] R. C. Gonzalez and R. E. Woods, "Digital image Processing", (2nd Edition), Pearson education, 2005.

[29] R. C. Gonzalez, R. E. Woods, and S.L. Eddins, "Digital Image Processing using MATLAB", Pearson Education, 2008 .

[30] N. C. Chauhan, M. V. Kartikeyan, and A. Mittal, "Soft Computing Methods for Microwave and Millimeterwave Design Problems", Studies in Computational Intelligence Series, Springer-Verlag, Berlin-Heidelberg, Germany, March 2012. 